\title{
Liquid chromatographic method for quantifying polyphenols in ciders by direct injection
}

\author{
Belén Suárez, Noemí Palacios, Natalia Fraga, Roberto Rodríguez* \\ Área de Tecnología de los Alimentos, Servicio Regional de Investigación y Desarrollo Agroalimentario (SERIDA), \\ 33300-Villaviciosa, Asturias, Spain
}

Received 18 November 2004; received in revised form 29 December 2004; accepted 14 January 2005

\begin{abstract}
An analytical method for the quantitative determination of the principal phenolic compounds (benzoic acids, hydroxycinnamic acids, 3phenylpropionic acids, flavanols, procyanidins, dihydrochalcones, quercetin glycosides) in ciders, which successfully employs a RP-HPLC and photodiode-array detection system without prior treatment of the sample, is described. Parameters usually examined in the method validation were evaluated. Good linearity was obtained with correlation coefficients exceeding 0.999 and the detection limits ranged from $0.07 \mathrm{mg} / \mathrm{L}$ ( $p$-hydroxybenzoic acid) to $2 \mathrm{mg} / \mathrm{L}$ (hydrocaffeic acid). Recoveries ranging between 90 and $104 \%$ and the reproducibility of the method was always $<8 \%$ (RSD). The method was applied to a set of commercial samples and the results obtained may be helpful to establish a phenolic profile in Asturian cider.

(C) 2005 Elsevier B.V. All rights reserved.
\end{abstract}

Keywords: Phenolic acids; Flavonoids; Cider; Direct injection; HPLC.

\section{Introduction}

Low molecular mass polyphenols have been exhaustively studied due to their contribution to the sensory quality (colour, taste, flavour) and their use for determining adulterations in apple derived products [1-5]. During the past decade the apple polyphenols have been subjected to a number of investigations due to their presence in human diet and their biological properties and benefit effects on health. Phenolic compounds seem to protect against cardiovascular disease and have certain potential anticarcinogenic properties due to their antioxidant activity and their function as free radical scavengers [6-9].

Polyphenols analysis, in cider and apple, is generally accomplished by reversed-phase high-performance liquid chromatography with UV-vis detection. However, the determination is usually preceded by several operations, such as extrac-

\footnotetext{
* Corresponding author. Tel.: +34 985890066; fax: +34 985891854 .

E-mail address: rrodriguez@serida.org (R. Rodríguez).
}

tion, purification and concentration, due to the complexity of analysis. Two methods for polyphenols fractionation have been used: liquid-liquid and solid-liquid extraction [10-13]. It should be noted that sample preparation is the time determining step of whole analytical procedure, which represents $2 / 3$ of the total analysis time, and it is the primary source of error differences in the results obtained by different laboratories [14]. Polyphenols are compounds very reactive and substantial changes in sample composition may occur due to: isomerizations under exposure to UV radiation or daylight, the oxidative transformation and hydrolysis phenomena as result of the extraction procedure [15-19]. Therefore, a direct injection of the sample could be considered as an alternative to simplify the analysis of phenolic compounds, and to prevent many errors and any polyphenols degradation during the sample handling [19-22].

In this paper, a reversed-phase HPLC method with diode array detection for the separation and quantitation of phenolic compounds in ciders, by direct injection, without any prior purification of sample, is described and validated. 


\section{Experimental}

\subsection{Reagents and standards}

Polyphenol standards were supplied as follows: (+)catechin, (-)epicatechin, phloridzin, tyrosol, catechol, benzoic acids (gallic acid, protocatechuic acid and $p$-hydroxybenzoic acid), hydroxycinnamic acids ( $p$-coumaric acid, caffeic acid, ferulic acid and chlorogenic acid), 3-phenylpropionic acids (hydrocoumaric acid, hydrocaffeic acid and hydroferulic acid,) by Sigma (St. Louis, MO, USA); and quercetin glycosides (hyperoside, isoquercitrin, avicularin, rutin and quercitrin) by Extrasyntèse (Genay, France). The phloretin$2^{\prime}$-xyloglucoside and procyanidins (B1, B2, B5, trimer C1, tetramer D and unknown trimer) were kindly furnished by Dr. A. Lea (Reading, UK). Water was purified on a Milli-Q system from Millipore (Bedford, MA, USA). Reagents and solvents were purchased from Panreac (Barcelona, Spain) and were of analytical or HPLC grade.

\subsection{Samples}

Eight Spanish ciders were purchased from local supermarkets of Asturias (Spain). Prior to HPLC analysis, the cider samples were degassed in an ultrasonic bath during $10 \mathrm{~min}$ in order to remove all carbon dioxide and filtered though a $0.45 \mu \mathrm{m}$ cellulose acetate membrane filter from Teknokroma (Barcelona, Spain).

\subsection{HPLC analysis}

Analyses were performed with a Waters system, equipped with a 717 automatic injector, provided with a column oven, two pumps (model 510), a diode array detector (model 996) and Millennium software v.2.1 data module. Separation of polyphenols was carried out on a reversed-phase Nucleosil $120 \mathrm{C}_{18}(250 \mathrm{~mm} \times 4.6 \mathrm{~mm}$ I.D., $3 \mu \mathrm{m})$ column from Teknokroma (Barcelona, Spain). The column was thermostated at $25^{\circ} \mathrm{C}$ and a flow rate of $0.8 \mathrm{~mL} / \mathrm{min}$ was used. The elution solvents were aqueous $2 \%$ acetic acid (solvent A) and $100 \%$ methanol (solvent B). The samples were eluted according to the following gradient: a linear step from 0 to $45 \%$ of solvent B in $55 \mathrm{~min}$ and a final isocratic step of $20 \mathrm{~min}$. Injection volume was $50 \mu \mathrm{L}$.

Identification of compounds was achieved by comparing their spectra and retention times with those of standards when available. Detection was performed at $313 \mathrm{~nm}$ for the hydroxycinnamic acids, at $355 \mathrm{~nm}$ for the flavonol glycosides and at $280 \mathrm{~nm}$ for the rest of phenolic compounds (benzoic and 3-phenylpropionic acids, flavanols, procyanidins and dihydrochalcones). The spectra were acquired from 200 to $400 \mathrm{~nm}$ with a sampling rate of 1.0 and the highest scanning resolution $(1 \mathrm{~nm})$.

Quantitation was performed according to an external standard method. For the compounds lacking of standards, or those which the amount at our disposal was too small, the quantification was achieved from similar compounds: thus, the procyanidins were quantified as procyanidin B1, the phloretin- $2^{\prime}$-xyloglucoside as phloridzin, the flavonol glycosides as quercitrin and the $p$-coumaroylquinic acid as $p$ coumaric acid.

\section{Results and discussion}

\subsection{Separation and identification}

Five families of phenols were taken as references for the analytical optimisation process in ciders: flavanols, hydroxycinnamic and 3-phenylpropionic acids, dihydrochalcones and flavonols.

Initial HPLC working conditions were selected on the basis of previously published works [12,23]. Two mobile phases
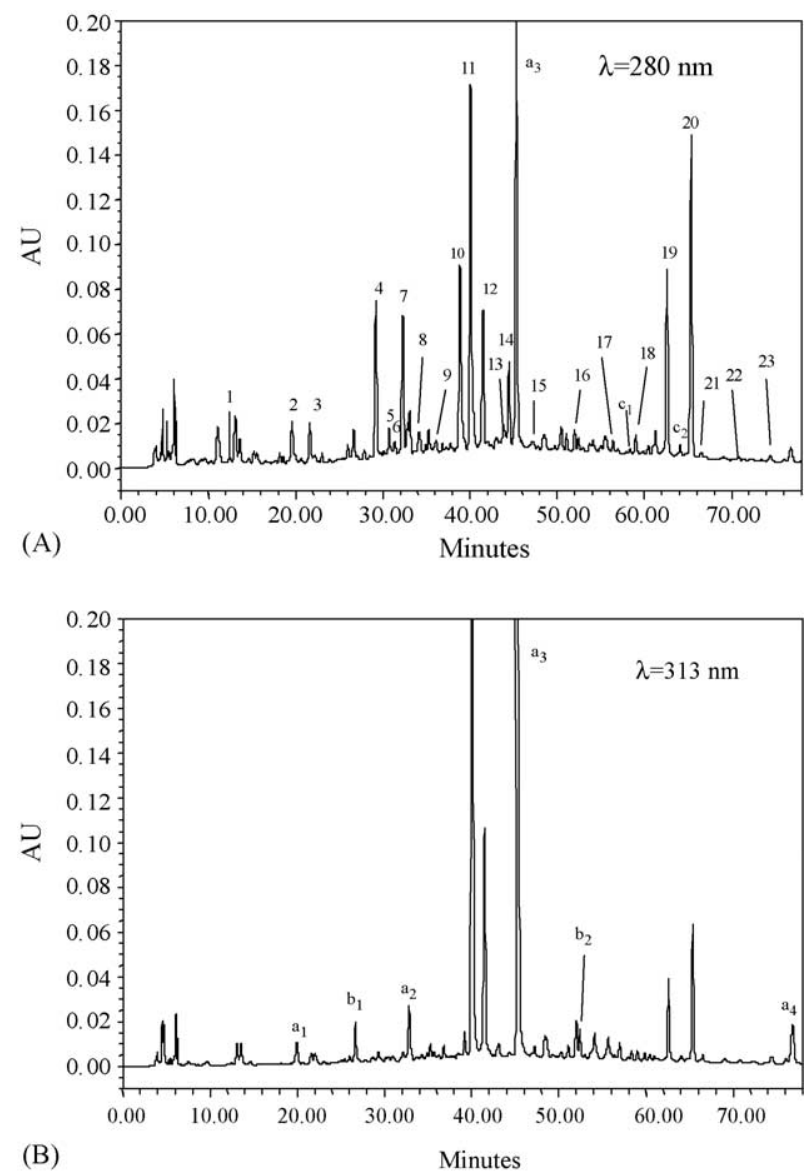

Fig. 1. Separation of phenolic compounds in cider at (A) $280 \mathrm{~nm}$ and (B) $313 \mathrm{~nm}$. Peaks: 1, gallic acid; 2, catechol; 3, protocatechuic acid; 4, tyrosol; 5, procyanidin B1; 6 , p-hydroxybenzoic acid; 7, hydrocaffeic acid; 8, (+)catechin; 9, unknown trimer; 10 , procyanidin $\mathrm{B} 2 ; 11$, chlorogenic acid; 12 , caffeic acid; 13 , tetramer $\mathrm{D} ; 14$, trimer $\mathrm{C} 1 ; 15$, hydroferulic acid; 16 , $p$-coumaric acid; 17 , ferulic acid; 18 , procyanidin B5; 19 , phloretin-2'xyloglucoside; 20, phloridzin; 21, hyperin; 22, avicularin; 23, quercitrin; $\mathrm{a}_{1}-\mathrm{a} 4, p$-coumaric derivatives $\left(\lambda_{\max }=312.0 \mathrm{~nm}\right) ; \mathrm{b}_{1}$ and $\mathrm{b}_{2}$, hydroxycinnamic derivates $\left(\lambda_{\max }=326.3 \mathrm{~nm}\right) ; c_{1}$ and $c_{2}$, phloretin derivates. 
were tested: $2 \mathrm{mM}$ phosphoric acid (solvent $\mathrm{A}$ )/methanol (solvent B) and $2 \%$ acetic acid (solvent A)/methanol (solvent $B$ ) elution gradient shapes, temperatures and flow rates in order to separate the maximum number of phenolic compounds.

The use of the phosphoric acid/methanol phase provided an overlapping of $p$-hydroxybenzoic and hydrocaffeic acids and worse resolution between procyanidin B2 and the unknown peak with hydroxycinnamic acid type spectra $\left(\lambda_{\max }=326 \mathrm{~nm}\right)$. Best resolution and faster separation were observed by using methanol/acetic acid as eluents. The optimized chromatographic conditions (Section 2.3) showed a good separation with values of selectivity factors $(\alpha)$ above 1.00 for flavan-3-ol (procyanidin B1, catechin, unknown trimer, procyanidin $\mathrm{B} 2$, trimer $\mathrm{C} 1$, tetramer D, epicatechin and procyanidin B5), phenolic acids (benzoic and $p$-hydroxycinnamic acids) and between phloridzin and flavonols. Under these optimized conditions coelution was only detected between rutin and isoquercitrin, while the rest of quercetin glycosides were properly separated. In fact, the problem of coelution between these compounds was reported by other authors [24].

A typical RP-HPLC chromatogram of a cider is shown in Fig. 1. In this cider sample a total of 23 phenolic compounds were identified by comparison of their retention times and
UV-vis spectra (catechol, tyrosol, nine phenolic acids, seven monomeric and oligomeric flavan-3-ols, two dihydrochalcones and three quercetin glycosides). The identified peaks were then confirmed by spiking samples with standard mixtures. Furthermore, the following compounds were tentatively identified via their spectral features and the review of literature data: peaks a1-a4 exhibited spectral characteristics similar to $p$-coumaric acid $\left(\lambda_{\max }=312.0 \mathrm{~nm}\right)$, the peaks b1 and b2 showed hydroxycinnamic acid type spectra $\left(\lambda_{\max }=326.0 \mathrm{~nm}\right.$, shoulder at $\left.297.7 \mathrm{~nm}\right)$ and peaks $\mathrm{c} 1$ and c2 had a phloridzin-like spectra. Probably, the peak a3 is $p$ coumaroylquinic the major phenolic acid quantified in cider together with chlorogenic acid $[25,26]$, and the peak a4 could be $p$-coumaric ethyl ester [27]. Other quinic esters of hydroxycinnamic acids have been characterized in apple derivates, such as chlorogenic acid and $p$-coumaroylquinic isomers and many derivatives of hydroxycinnamics acid and glucose can also be found in small quantities as $p$-coumaroylglucose and feruloylglucose [24,28]. With regard to the phloretin derivatives (peaks $\mathrm{c} 1$ and $\mathrm{c} 2$ ), the 3-hydroxyphloridzin has been identified in apple pomace and its chemical structure was established by NMR [29] and also tentatively identified by HPLC-MS in Basque apple cider [30]. Another dihydrochalcone was recently described in Golden Delicious apples [31].

Table 1

Analytical characteristics of the calibration graphs and recoveries of phenolic compounds from cider

\begin{tabular}{|c|c|c|c|c|c|c|c|}
\hline \multirow[t]{2}{*}{ Compound } & \multicolumn{4}{|c|}{ Calibration curve $(n=15)$} & \multirow[t]{2}{*}{$\mathrm{LOD}(\mathrm{mg} / \mathrm{L})$} & \multirow{2}{*}{$\begin{array}{l}\text { Mean recovery } \\
(\%)(n=6)\end{array}$} & \multirow[t]{2}{*}{$\operatorname{RSD}^{\mathrm{a}}(\%)$} \\
\hline & Linear range $(\mathrm{mg} / \mathrm{L})$ & Slope & Intercept & Correlation coefficient & & & \\
\hline Catechol & $3.0-30.3$ & 86379.9 & -11850.0 & 0.9999 & 0.3 & 92 & 3.0 \\
\hline Tyrosol & $2.5-50.0$ & 35207.0 & -8444.6 & 0.9999 & 0.5 & 91 & 2.9 \\
\hline \multicolumn{8}{|l|}{ Benzoic acids } \\
\hline Gallic & $0.3-5.5$ & 170572.6 & -10366.7 & 0.9999 & 0.09 & 97 & 6.3 \\
\hline Protocatechuic & $0.8-15.9$ & 97787.7 & -12904.1 & 0.9999 & 0.2 & 93 & 7.6 \\
\hline$p$-Hydroxybenzoic & $0.6-6.4$ & 189852.0 & -6629.5 & 0.9999 & 0.07 & 92 & 5.0 \\
\hline \multicolumn{8}{|l|}{$\begin{array}{l}\text { Hydroxycinnamic } \\
\text { acids }\end{array}$} \\
\hline Hydrocaffeic & $10.7-214.0$ & 54308.1 & -59043.7 & 0.9999 & 2 & 94 & 2.6 \\
\hline Chlorogenic & $3.9-39.0$ & 158849.1 & -40110.0 & 0.9996 & 0.6 & 104 & 3.7 \\
\hline Caffeic & $4.2-21.0$ & 325901.0 & -75401.0 & 0.9999 & 0.5 & 92 & 1.0 \\
\hline Hydrocoumaric & $2.6-51.5$ & 38436.2 & 1763.9 & 0.9997 & 0.5 & 93 & 2.6 \\
\hline Hydroferulic & $0.5-8.6$ & 55459.3 & -5985.6 & 0.9998 & 0.2 & 93 & 2.5 \\
\hline$p$-Coumaric & $3.8-38.0$ & 474518.0 & -139986.0 & 0.9997 & 0.5 & 90 & 2.6 \\
\hline Ferulic & $0.5-10.2$ & 359849.5 & -18505.1 & 0.9999 & 0.2 & 95 & 3.2 \\
\hline \multicolumn{8}{|l|}{$\begin{array}{l}\text { Procyanidin and } \\
\text { flavanols }\end{array}$} \\
\hline Catechin & $1.1-22.1$ & 44382.2 & -5025.5 & 0.9996 & 0.4 & 93 & 7.7 \\
\hline Epicatechin & $1.6-32.1$ & 45832.9 & -15415.6 & 0.9994 & 0.8 & 99 & 7.4 \\
\hline Procyanidin B1 & $1.2-74.1$ & 37013.6 & -380.6 & 0.9997 & 0.3 & 94 & 3.8 \\
\hline \multicolumn{8}{|l|}{ Dihydrochalcone } \\
\hline Phloridzin & $3.8-61.7$ & 141371.1 & -37745.3 & 0.9999 & 0.6 & 94 & 3.2 \\
\hline \multicolumn{8}{|l|}{ Flavonol } \\
\hline Quercitrin & $0.6-9.9$ & 101814.7 & -4771.8 & 0.9994 & 0.2 & 93 & 3.7 \\
\hline
\end{tabular}

LOD: Limit of detection.

${ }^{a}$ RSD of mean recovery. 


\subsection{Validation procedure}

To check the linearity of the response of detector, a linear regression analysis of absolute areas versus concentration of the phenolic compounds was used. The linearity was determined by the square correlation coefficients of the calibration curves generated by three repeated injections of standard solutions at five concentrations levels, with concentrations included the range expected in real samples. The limit of detection was estimated from the residuals of calibration graph [32]. Calibration parameters are shown in Table 1. All the compounds showed a good linearity with regression coefficients $>0.9990$ the limit of detection (LOD) ranging from $0.07 \mathrm{mg} / \mathrm{L}$ for $p$-hydroxybenzoic to $2 \mathrm{mg} / \mathrm{L}$ for hydrocaffeic acid. These results suggest that the proposed HPLC method is sufficiently sensitive for the determination of phenolic compounds in apple derivates, in accordance with previous reports $[31,33,34]$.
Recovery experiments were performed in order to study the accuracy of the method. Know amounts of pure standards were added to a sample cider, at three different concentrations levels, in duplicate (Table 1). Recoveries ranged between 90 and $104 \%$ and these values testify the accuracy of the proposed method.

Precision was studied in a real sample in two ways: retention times and peak areas. The repeatability of peak areas and retention times were calculated by the RSD of five injections carried out on the same day. The RSD for the retention time of all peaks was $<0.7 \%$ and the coefficient of variation for the peak areas was $<2 \%$. The reproducibility of the method was evaluated during recovery experiments and the RSD were always $<8 \%$ (Table 1 ).

To improve the selectivity, different wavelengths were, used for quantify the phenolic compounds: $313 \mathrm{~nm}$ for the hydroxycinnamic acids, $355 \mathrm{~nm}$ for quercetin glycosides and 280 for the rest of compounds. Additionally, the peak pu-

Table 2

Contents of phenolic compounds in commercial ciders

\begin{tabular}{|c|c|c|c|c|c|c|c|c|c|}
\hline \multirow[t]{2}{*}{ Compound } & \multicolumn{9}{|c|}{ Content $^{\mathrm{a}}(\mathrm{mg} / \mathrm{L})$} \\
\hline & $t_{\mathrm{R}}(\min )$ & Cider 1 & Cider 2 & Cider 3 & Cider 4 & Cider 5 & Cider 6 & Cider 7 & Cider 8 \\
\hline Gallic acid & 12.56 & nd & nd & 1.2 & 0.4 & 0.1 & 0.6 & nd & nd \\
\hline Catechol & 19.58 & 4.3 & 2.7 & 3.9 & 1.8 & 3.0 & 7.2 & nd & 3.2 \\
\hline Protocatechuic acid & 21.62 & 0.5 & 0.6 & 1.5 & 1.3 & 1.8 & nd & 3.0 & 3.6 \\
\hline Tyrosol & 29.22 & 15.0 & 31.6 & 28.8 & 35.1 & 41.5 & 22.0 & 23.1 & 30.5 \\
\hline Procyanidin B1 & 30.74 & 5.8 & 5.6 & 15.5 & 11.4 & 4.0 & 13.7 & 7.9 & 16.6 \\
\hline$p$-Hydroxybenzoic & 31.40 & 1.2 & 1.1 & 0.8 & 1.6 & 0.9 & 1.6 & 1.4 & 1.3 \\
\hline Hydrocaffeic acid & 32.29 & 68.4 & 74.7 & 93.5 & 97.3 & 94.9 & 110.5 & 55.8 & 110.5 \\
\hline Catechin & 34.14 & 1.6 & 1.1 & 1.3 & 2.0 & 0.8 & 2.1 & 2.7 & 7.9 \\
\hline Unknown trimer ${ }^{b}$ & 36.12 & 5.0 & 2.9 & 6.8 & 6.3 & 2.4 & 7.1 & 3.6 & 5.1 \\
\hline Procyanidin $\mathrm{B} 2^{\mathrm{b}}$ & 38.84 & 36.0 & 33.9 & 61.4 & 43.9 & 23.6 & 65.1 & 31.6 & 66.8 \\
\hline Chlorogenic acid & 40.07 & nd & nd & nd & nd & nd & nd & 21.5 & nd \\
\hline Caffeic acid & 41.48 & nd & nd & nd & nd & nd & nd & 10.3 & 12.3 \\
\hline Hydrocoumaric acid & 42.18 & 42.6 & 19.5 & 48.7 & 44.3 & 23.3 & 32.9 & 15.9 & 8.8 \\
\hline Tetramer $\mathrm{D}^{\mathrm{b}}$ & 43.00 & 5.5 & 4.3 & 11.4 & 6.9 & 2.0 & 10.9 & 4.7 & nd \\
\hline Trimer $\mathrm{C} 1^{\mathrm{b}}$ & 43.82 & 12.4 & 12.0 & 14.6 & 11.7 & 12.3 & 17.2 & 12.7 & nd \\
\hline Epicatechin & 44.49 & nd & nd & 6.6 & nd & 4.1 & 3.2 & 9.9 & 30.3 \\
\hline$p$-Coumaroylquinic ${ }^{c}$ & 45.31 & 13.6 & 14.6 & 20.9 & 15.8 & 10.6 & 22.0 & 12.5 & 21.3 \\
\hline Hydroferulic acid & 47.82 & 4.0 & nd & 1.3 & nd & 3.9 & 1.7 & 2.9 & 2.9 \\
\hline$p$-Coumaric acid & 52.43 & nd & nd & nd & nd & 0.8 & nd & 0.7 & nd \\
\hline Unknown procyanidin ${ }^{\mathrm{b}}$ & 55.00 & 4.0 & 4.5 & 4.6 & 6.7 & 3.2 & nd & 9.4 & nd \\
\hline Ferulic acid & 56.41 & 0.8 & 0.3 & 0.4 & 0.4 & nd & nd & 1.6 & 1.1 \\
\hline Unknown phloretin derivative $(1)^{\mathrm{d}}$ & 58.36 & 1.6 & 1.2 & 2.2 & 1.1 & 1.5 & 2.6 & 0.6 & 2.0 \\
\hline Procyanidin $\mathrm{B}^{\mathrm{b}}$ & 59.01 & 3.1 & 5.7 & 7.0 & 2.6 & 10.4 & 9.5 & 6.1 & 2.6 \\
\hline Phloretin $2^{\prime}$-xyloglucoside ${ }^{\mathrm{d}}$ & 62.59 & 7.4 & 2.0 & 9.3 & 25.4 & 4.6 & 5.8 & 36.5 & 28.5 \\
\hline Unknown phloretin derivative $(2)^{\mathrm{d}}$ & 64.10 & nd & nd & nd & 0.8 & 0.8 & nd & 1.8 & 1.4 \\
\hline Phloridzin & 65.36 & 24.8 & 19.6 & 38.0 & 27.5 & 21.6 & 53.7 & 8.9 & 34.1 \\
\hline Hyperin $^{\mathrm{e}}$ & 66.52 & 2.0 & 1.3 & 3.8 & 1.6 & 0.5 & 6.8 & nd & 1.5 \\
\hline Rutin + isoquercitrin ${ }^{\mathrm{e}}$ & 67.58 & 0.3 & 0.2 & 0.6 & nd & nd & nd & 0.6 & nd \\
\hline Unknown flavonol $^{\mathrm{e}}$ & 68.75 & 0.5 & 0.3 & 0.9 & 0.4 & nd & 1.8 & nd & 0.4 \\
\hline Avicularin $^{\mathrm{e}}$ & 70.50 & 1.0 & 0.5 & 1.1 & 0.6 & 0.1 & 2.4 & 0.2 & 0.9 \\
\hline Quercitrin & 74.42 & 2.2 & 2.5 & 4.3 & 2.7 & 2.7 & 6.3 & 2.4 & 3.1 \\
\hline Unknown $p$-coumaric acid derivative ${ }^{c}$ & 76.26 & 0.6 & 0.6 & 1.0 & 0.7 & 1.0 & 1.0 & 0.7 & 0.1 \\
\hline
\end{tabular}

nd: not detected; LOD: Limit of detection; $t_{\mathrm{R}}$ : retention time.

a Mean values for three injections.

b Quantified as procyanidin B1.

c Quantified as $p$-coumaric acid.

d Quantified as phloridzin.

e Quantified as quercitrin. 
rity was checked by means of the PDA Millenium software spectral contrast facilities.

\subsection{Cider sample analysis}

The optimized and validated method was applied to the analysis of eight different commercial ciders from Asturias (Spain). Table 2 lists the range of concentrations of the phenolic compounds determined.

The phenolic compounds found in ciders varied quantitatively with the nature of raw materials (apple varieties, cultivation conditions) and the cidermaking procedures [1,26,35-38]. In all the ciders analysed the major phenolic compound was hydrocaffeic acid with levels that represent a $30 \%$ of the total polyphenols assayed. This compound is probably generated by hydrolysis of caffeic acid derivatives by microorganisms during fermentation and postfermentation steps $[25,40]$. In contrast, caffeic acid was detected in two ciders only, while chlorogenic acid was found in one of the samples. The procyanidin B2 was always the major compound among the flavonoids, with values that varied from $23.6 \mathrm{mg} / \mathrm{L}(24 \%)$ to $66.8 \mathrm{mg} / \mathrm{L}(33 \%)$. Phloridzin and phloretin- $2^{\prime}$-xyloglucoside were found in all samples analysed, confirming the usefulness of these compounds as chemical markers for apple derivatives [5]. Likewise, flavonols should be considered as a minor class, because they represent less than $5 \%$ of total phenolic compounds. An unknown flavonol $\left(t_{\mathrm{R}}=68.75 \mathrm{~min}\right)$ detected could correspond to reynoutrin $[41,42]$ or a quercetin pentoside reported by Shieber et al. [42] and Lommen et al. [43]. Tyrosol, which is produced during fermentation by deamination of the amino acid tyrosine, was present in all samples analysed.

Spanish natural cider presents differences [26,38,44] when compared to French or English ones, which are reflected in the sensorial properties and chemical compositions of the product. These differences are attributable to multitude of variables such as apple variety used, cultivation conditions, climatology, processed and fermentation technology used and maturation and aging. Spanish natural cider is dry and acidic with a balanced aroma described by fresh, while French ciders are characterised by sweeter, tannic and applelike flavours, whereas English ciders are carbonated products, with varying degrees of sweetness and an alcoholic proof, being and the flavour generally smoother compared with that described 30 years ago $[25,26,45,46]$.

\section{Conclusion}

A method for accurately quantifying the phenolic compounds in cider using a direct injection has been successfully developed. The analytical method described is suitable for routine analysis of phenolic acids and flavonoids in a single chromatogram avoiding the extra time required in purification steps and the possible generation of artefacts or degradation of sample during the extraction step.

\section{Acknowledgments}

The authors are indebted to the Comisión Interministerial de Ciencia y Tecnología (CICYT AGL2001-0713) and the Government of the Principado de Asturias for financial support.

\section{References}

[1] A.G.H. Lea, Arnold, J. Sci. Food Agric. 29 (1978) 478.

[2] A.G.H. Lea, Fluess. Obst. Heft. 8 (1984) 1.

[3] W. Oleszek, C.Y. Lee, A.W. Jaworski, K.R. Price, J. Agric. Food Chem. 26 (1988) 430.

[4] M.J. Amiot, M. Tacchini, S. Aubert, J. Nicolas, J. Food Sci. 57 (1992) 958.

[5] G.A. Spanos, R.E. Wrolstad, J. Agric. Food Chem. 40 (1992) 1478.

[6] M.G.L. Hertog, P.C.H. Hollman, B. Van de Putte, J. Agric. Food Chem. 41 (1993) 1242.

[7] M.S. Dupont, R.N. Bennet, F.A. Mellon, G. Williamson, J. Nutr. 132 (2002) 172.

[8] H. Leontowicz, S. Gorinstein, A. Lojej, M. Leontowicz, M. Ciz, R. Soliva-Fortuny, Y.S. Park, S.T. Jung, S. Trakhtenberg, O. MartinBelloso, J. Nutr. Biochem. 13 (2002) 603.

[9] F. Chinnici, A. Bendini, A. Gaiani, C. Riponi, J. Agric. Food Chem. 52 (2004) 4684.

[10] E. Delage, G. Bohuon, A. Baron, J.F. Drilleau, J. Agric. Food. Chem. 555 (1991) 125.

[11] B. Suárez, J. Santamaría, J.J. Mangas, D. Blanco, J. Agric. Food Chem. 42 (1994) 2732.

[12] B. Suárez, A. Picinelli, J.J. Mangas, J. Chromatogr. A 727 (1996) 203.

[13] R.M. Alonso-Salces, E. Korta, A. Barranco, L.A. Berrueta, B. Gallo, F. Vicente, J. Chromatogr. A 933 (2001) 37.

[14] R.E. Majors, LC-GC Int. 4 (1991) 10.

[15] B. Monties, Ann. Physiol. Vég. 8 (1966) 101.

[16] G. Kahnt, Phytochemistry 6 (1967) 84.

[17] M.N. Clifford, B. Kellard, G.G. Birch, Food Chem. 33 (1989) 115.

[18] S. Guyot, N. Marnet, D. Laraba, P. Sanoner, J.F. Drilleau, J. Agric. Food Chem. 46 (1998) 1698.

[19] J.P. Roggero, S. Coen, P. Archier, J. Liq. Chromatogr. 13 (1990) 2593.

[20] G. Achilli, G.P. Cellerino, P.H. Gamache, J. Chromatogr. 632 (1993) 111.

[21] R.M. Lamuela-Raventón, A.L. Waterhouse, Am. J. Enol. Vitic. 45 (1994) 1 .

[22] B. Suárez, A. Picinelli, J. Moreno, J.J. Mangas, J. Sci. Food Agric. 78 (1998) 461.

[23] A. Escarpa, M.C. González, J. Chromatogr. A 830 (1999) 301.

[24] J. Pérez-Ilzarbe, T. Hernández, I. Estrella, Z. Lebensm Unters Forsch 192 (1991) 552.

[25] G.C. Whiting, R.A. Coggins, J. Sci. Food Agric. 26 (1975) 1833.

[26] R.M. Alonso-Salces, S. Guyot, C. Herrero, L.A. Berrueta, J.F. Drilleau, B. Gallo, F. Vicente, Anal. Bioanal. Chem. 379 (2004) 464.

[27] C. Garcia-Parrilla, F.J. Heredia, A.M. Troncoso, J. Liquid. Chromatogr. Rel. Technol. 19 (1996) 247.

[28] J.J. Macheix, A. Fleuriet, J. Billot, Fuit Phenolics, CRC Press, Boca Ratón, FL, 1990, p. 113.

[29] Y. Lu, L.Y. Foo, Food Chem. 59 (1997) 184.

[30] R.M. Alonso-Salces, K. Ndjoko, E.F. Queiroz, J.R. Ioset, K. Hostettmann, L.A. Berrueta, B. Gallo, F. Vicente, J. Chromatogr. A 1046 (2004) 89.

[31] F. Chinnici, A. Gaiani, N. Natali, C. Riponi, S. Galassi, J. Agric. Food Chem. 52 (2004) 3.

[32] J.M. Millar, Analyst 116 (1991) 3. 
[33] A. Ecarpa, M.C. González, J. Chromatogr. A 823 (1998) 331.

[34] D. Blanco, N. Fraga, J.J. Mangas, Anal. Chim. Acta 426 (2001) 111.

[35] A.G.H. Lea, C.F. Timberlake, J. Sci. Food Agric. 29 (1978) 484.

[36] A. Picinelli, B. Suárez, J.J. Mangas, Z. Lebensm Unters Forsch A 204 (1997) 48.

[37] J.J. Mangas, R. Rodríguez, B. Suárez, A. Picinelli, E. Dapena, J. Agric. Food Chem. 47 (1999) 4046.

[38] B. Suárez, A. Piccinelli, Recent Res. Dev. Agric. Food Chem. 5 (2001) 1.

[40] F.W. Beech, J.G. Carr, in: A.H. Rose (Ed.), Alcoholic Beverages, Academic Press, London, 1977, p. 188.
[41] K.R. Price, T. Prosser, A.M.F. Richetin, M.J.C. Rhodes, Food Chem 66 (1999) 489.

[42] A. Shieber, P. Keller, R. Carle, J. Chromatogr. A 910 (2001) 265.

[43] A. Lommen, M. Godejohan, D.P. Venema, P.C. Hollman, M. Spraul, Anal. Chem. 72 (2000) 1793.

[44] A. Picinelli, B. Suárez, J. Moreno, R. Rodríguez, L. Caso-García, J.J. Mangas, J. Agric. Food Chem. 48 (2000) 3997.

[45] A.G.H. Lea, C.F. Timberlake, J. Sci. Food. Agric. 25 (1974) 1537.

[46] A.G.H. Lea, in: A.G.H. Lea, J.R. Piggot (Eds.), Fermented Beverage Production, Blackie Academic \& Professional, London, 1995, p. 65. 